\section{Parkinson-Therapie: Her mit den Vitaminen!}

\author{
Die Langzeitgabe von Levodopa zur Therapie des Parkinson-Syndroms \\ hat auch metabolische Konsequenzen, die den neuronalen Zelltod fördern \\ können. Deshalb empfehlen einige Experten ihren Patienten regelhaft die \\ Vitaminsubstitution.
}

$\mathrm{D}$ iskutiert wird schon länger die Gabe von B-Vitaminen bei entsprechendem Mangel während einer DuodopaTherapie [1]. Doch auch bei oraler Applikationsform von Levodopa macht die Vitaminsubstitution bei Langzeittherapie laut Professor Thomas Müller, Klinik für Neurologie am St. Joseph Krankenhaus, Berlin-Weißensee, Sinn. Postuliert wird, dass Levodopa über zwei Mechanismen den neuronalen Zelltod fördern kann: Durch die Folgen des Abbaus durch Methylierung und über freie Radikale. Beim Abbau von Levodopa zu 3-O-Methyldopa durch Methylierung mit der Catechol-O-Methyltransferase (COMT) wird die Homocysteinsynthese unterstützt, so die Hypothese. Eine bei dauerhafter Levodopa-Gabe entstehende Hyperhomocystämie erhöht aber den oxidativen Stress. Gleichzeitig nimmt Methionin ab, was ebenfalls zu mehr oxidativem Stress und einer mitochondrialen Dysfunktion führt, ergänzte Müller. Die bei oxidativem Stress frei werdenden reaktiven Sauerstoffspezies können aber über Lipidperoxidation, Proteinoxidation und DNA-Schädigung den Zelltod fördern. Bestätigt wird der postulierte Mechanismus durch den Effekt bei zusätzlicher Gabe eines COMTHemmers, der die Methylierung verhindert. So stieg der Homocysteinspiegel bei Patienten mit Parkinson-Syndrom an, wenn sie eine Therapie mit retardier-

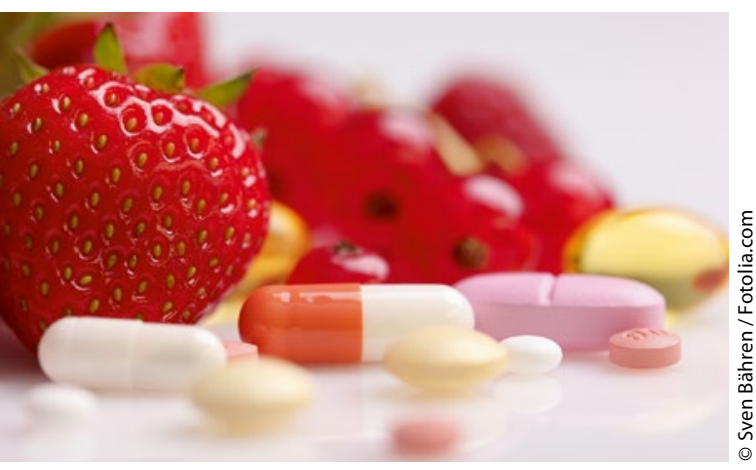

tem Levodopa/Carbidopa (200 mg) erhielten, nicht aber, wenn sie eine Kombination von $150 \mathrm{mg}$ Levodopa/Carbidopa und zusätzlich Entacapon einnahmen. Dabei blieb die Pharmakokinetik von Levodopa in beiden Fällen gleich [2].

\section{Rationale für Vitamine}

Der Anreicherung von Homocystein, das eine relativ lange Halbwertszeit hat, kann durch die Gabe von Methylgruppendonatoren entgegengewirkt werden. Die Methylgruppenübertragung führt dann zu einer Verstoffwechselung von Homocystein zu Methionin. Als Überträger kommen Vitamin B6, Vitamin B12 und Folsäure infrage. Müller konnte bereits zeigen, dass eine solche Vitamingabe tatsächlich einen weiteren Homocysteinanstieg bei Levodopa-Langzeittherapie verhindert [3]. Außerdem wird Homocystein über die Vitamin-B6abhängige irreversible Synthese zu Cystein abgebaut, das wiederum wesentlich ist für die Glutathionsynthese. Glutathion ist aber das wirksamste System des Körpers zur Eliminierung freier Radikale, betonte Müller. Ein hoher Glutathionverbrauch und ein niedriger Spiegel des freien Cysteinyl-Glycins und Cysteins sind daher ein Zeichen für oxidativen Stress. Tatsächlich zeigt sich bei Gabe von $200 \mathrm{mg}$ Levodopa/Carbidopa ein Cysteinabfall [4].

Sein Fazit für die Praxis. „Empfehlen Sie Ihren Patienten mit Parkinson-Syndrom bei Therapie mit Levodopa oder Duodopa ein Vitamin-B-Mischpräparat oder $5 \mathrm{mg}$ Folsäure pro Tag wie in der Schwangerschaft. Dass damit auch Polyneuropathien aufgrund eines B-VitaminMangels verschwinden, ist ein weiterer günstiger Effekt."

\section{Vorsicht Gewichtsreduktion!}

Aber nicht nur ein Vitaminmangel kann für Patienten mit Parkinson-Syndrom problematisch sein. Es besteht eine Neigung zur Gewichtsabnahme, die bei der chronischen Erkrankung zusätzlich zur Morbidität und Mortalität beitragen kann. Dabei scheint eine überdurchschnittliche Gewichtsabnahme sogar eine prämotorische Manifestation eines Parkinson-Syndroms zu sein und aufzutreten, obwohl die Patienten sich bemühen, mehr Energie aufzunehmen [5]. Wahrscheinlich liegen dem veränderte intestinale Resorptionseigenschaften zugrunde, erläuterte Professor Fabian Klostermann, Klinik für Neurologie der Charité in Berlin. Hauptgrund könnte eine krankheitsbedingte vegetative Synukleopathie sein. Tatsächlich kumulieren Lewy-Körperchen in enterischen Nervenzellen wie später im Gehirn. Erst in späteren Stadien der Erkrankung scheint auch ein erhöhter krankheitsbedingter Energieverbrauch eine Rolle für die weitere Gewichtsabnahme zu spielen.

Aber auch iatrogene Effekte auf den Ernährungsstatus spielen eine Rolle: Hohe Levodopa-Dosen scheinen den krankheitsbedingten Trend zur Gewichtsreduktion zu verstärken. In einer Studie war die tägliche Levodopa- oder dopaminerge Dosis pro kg Körpergewicht negativ korreliert mit dem Body Mass Index [6]. Als weiteren Beleg führte Klostermann an, dass nach einer Operation zur tiefen Hirnstimulation oft eine deutliche Gewichtszunahme zu beobachten ist, die neben den antidyskinetischen Wirkungen auch durch den Levodopa-einsparenden Effekt verursacht sein könnte. Er empfiehlt das Monitoring von Gewicht, klinischen Zeichen der Neuropathie (als Zeichen des B-Vitamin-Mangels) sowie der B-Vitamine-, Folsäure- und Homocysteinspiegel, um relevanten Folgeproblemen der Levodopa-Therapie präventiv entgegenwirken zu können.

Friederike Klein

1. Jost W et al. Akt Neurol 2013; 40: 1 -3

2. Müller T, Muhlack S. J Neural Transm. 2009; 116: $1253-6$

3. Müller T et al. Clin Neuropharmacol 2013, 36: $52-44$

4. Müller T, Muhlack S. Neurosci Lett. 2012; 521: $37-9$

5. Chen H Ann Neurol 2003; 5: 676-9

6. Bachmann et al. Eur J Neurol 2009; 16: $895-901$

Mini-Symposium: „Levodopa - ein Revival?", 26.9.2015, 88. Kongress der Deutschen Gesellschaft für Neurologie, Düsseldorf, 23. - 26.9.2015 